\title{
Enhanced Channel Assignment and Load Distribution in IEEE 802.11 WLANs
}

\author{
Dr. Hussain Al-Rizzo ${ }^{1}$, Mohamad Haidar ${ }^{1}$, Dr. Robert Akl ${ }^{2}$, Dr. Yupo Chan ${ }^{1}$ \\ ${ }^{1}$ Department of Systems Engineering, Donaghey College of Information Science and Systems Engineering, \\ University of Arkansas at Little Rock, Little Rock, AR 72208-1044 \\ hmalrizzo, mxhaidar, yxchan@ualr.edu \\ ${ }^{2}$ Department of Computer Science and Engineering, University of North Texas, TX 76207-7102, \\ rakl@cse.unt.edu
}

\begin{abstract}
An algorithm to reduce congestion and balance users' load in IEEE $802.11 \mathrm{~b} / \mathrm{g}$ Wireless Local Area Networks (WLANs) is presented, which takes into account overlapping channel interference between Access Points (APs) and the signal-to-interference ratio (SIR) experienced by the users. After finding the best channel assignment at the APs, the algorithm then finds the Most Congested Access Point (MCAP). It reexamines the users' association with APs by minimizing the congestion at the MCAP. Simulation results show that the proposed algorithm is capable of significantly reducing the overall congestion in the WLAN while mitigating channel interference. Our algorithm has also been shown to be scalable and it performs well for networks of different topologies.
\end{abstract}

\section{Keywords-MCAP; WLAN; AP; Interference}

\section{INTRODUCTION}

Channel assignment and load balancing in IEEE 802.11 WLANs have attracted attention of both industry and academic community $[1,2,3,4,6]$. In $[1,2]$ an approach is proposed to minimize AP congestion using an Integer Linear Program (ILP). The objective is to mitigate the most congested APs, or the most popular hot spots in a WLAN. The model proposed in $[1,2]$ assumed the transmitted power by APs fixed at all times. Moreover, the associated ILP did not take into account overlapping channel interferences among APs.

In [3], the authors proposed a load balancing procedure that allows a wireless station to join an AP depending on the number of stations already associated with it, the mean Received Signal Strength Indicator (RSSI), and other link-quality measures. Each AP updates the number of associated stations and its mean RSSI continuously in each beacon or probe response frame. The algorithm performed well under traffic consisting of three APs and 30 users. However, the algorithm was neither tested for larger networks nor took interferences between APs into account.

The authors in [4] proposed a load-balancing scheme for overlapping wireless cells. Load Balancing
Agents (LBA) running in each AP broadcast periodically the load of APs through the Ethernet backbone and determines whether the AP is overloaded. In overloaded APs, users will be dissociated with the corresponding AP and reassociated with under-loaded APs. The scheme did not take into account Signal-to-Interference Ratio (SIR) from neighboring APs.

In [5], the authors employed an indoor wireless testbed to emulate the performance of real-world networks of one AP and one interferer. Although they discussed heavily the throughput-degradation effect of SIR and Signal-to-Noise-Ratio (SNR), they only tested one kind of interference that is generated by microwave ovens operating at a center frequency of $2.45 \mathrm{GHz}$.

Finally, the authors in [6] presented a cell breathing technique that performs load balancing to improve Quality of Service (QoS) in real-time applications. Nonetheless, the authors decreased power level at congested APs, causing the cell size to shrink, thus reducing the number of active users associated with the affected APs. Interference between neighboring APs is not taken into consideration. This might cause a newly dissociated user to associate with a neighboring AP that meets a certain RSSI threshold but only at a high interference level.

In this paper, we propose to reduce the load at the MCAP while using the SIR to configure the initial user-association. The initial association is then optimized using the software LINGO [7]. This way, the idea of reducing the MCAPs is intuitive and easy to implement. Reducing the load on the MCAP is implemented by assigning certain users to less congested APs, leading to a more balanced load. We will show that the performance of our algorithm outperforms those described in [1, 2] for various network topologies. The remainder of the paper is organized as follows. The load balancing algorithm is presented in section II. In section III, numerical results are presented, and finally section IV concludes the paper.

\section{LOAD BALANCING ALgORITHM}

We consider a WLAN that consists of a grid of $M$ 
APs distributed in a single-floor indoor environment. A set of $N$ users roams randomly, each seeking to associate with an AP. Each user $i$ is defined by its data rate, $U_{i}$ bits/s, randomly ranging between 1.0 $\mathrm{Mb} / \mathrm{s}$ to $7.0 \mathrm{Mb} / \mathrm{s}$.

A No-Line-of-Sight (NLOS) commercial Path Loss model has been used to determine the power level at each user's location, as described in (1) [5]:

$$
P L\left(d_{i j}\right)=P L+29.4 \log _{10}\left(\frac{d_{i j}}{d_{0}}\right)+6.1 x_{\alpha} \log _{10}\left(\frac{d_{i j}}{d_{0}}\right)+24 y+1.3 x_{s} y,
$$

where $P L_{0}$ is the free space path loss, $d_{i j}$ is the distance between user $i$ and $\operatorname{AP} j, d_{0}<d$ is the reference distance at 1 meter (indoor), and $x_{\alpha}, x_{s}$, and $y$ are mutually independent Gaussian random variables of zero mean and unit variance.

Given the AP's location, a channel-assignment algorithm [8] was used to assign channels to the available grid of APs using the following algorithm:

$\min _{F_{i}} \sum_{j=1}^{Q_{i}} I_{i j}$,

subject to $\quad I_{i j}=\frac{w_{i j} P_{j}}{P L\left(d_{i j}\right)}$,

$$
w_{i j}=\left\{\begin{array}{cl}
1-\frac{\left|F_{i}-F_{j}\right|}{5} & \text { if } w_{i j} \geq 0 \\
0 & \text { otherwise }
\end{array}\right.
$$

for $j \in \mathbf{A}_{\mathbf{i}}$, for $F_{i} \in\{1, \ldots, K\}$,

Here $F_{i}$ is the channel assigned to $\mathrm{AP} i, Q_{i}$ is the cardinality of $\mathbf{A}_{\mathbf{i}}$, the set of the APs next to AP $i, I_{i j}$ is the interference that $\mathrm{AP} j$ causes on $\mathrm{AP} i, w_{i j}$ is the interference factor due to overlapping channels, $P_{j}$ is the transmit power level of AP $j, P L$ is an exogenously computed function that captures the attenuation loss based on the propagation model described in Eqn (1), and $d_{i j}$ is the distance between AP $i$ and AP $j . K$ is the total number of channels available in IEEE $802.11 \mathrm{~b} / \mathrm{g}$.

Once the channels are assigned to the AP's, SIR from all AP's can be calculated at each user in the network using the following equation.

$$
\begin{aligned}
& S I R=\frac{P_{n}}{I_{n^{\prime}}}, \\
& \text { for } n \in\{1, \ldots, M\}, \\
& n^{\prime} \in\{1, \ldots, M\} \text { and } n^{\prime} \neq n,
\end{aligned}
$$

where $P_{n}$ is the desired signal received by a given user from its respective AP, $I_{n}$, is the interference caused by neighboring AP's on that user.
Once the power received by a user from an AP exceeds the receiver sensitivity threshold of $-85 \mathrm{dBm}$ and its SIR $>0$, that user becomes a potential candidate for association with that AP. Thus, a single user can be a potential candidate for multiple APs.

Using another mathematical programming model, we aim to redistribute the users' associations in order to minimize the overall congestion in the network. This is done by first identifying the MCAP. Correspondingly, an objective function is defined as minimizing congestion at the MCAP. This is done with the additional constraint that each user must be associated with one-and-only-one AP at any time.

The congestion factor at each AP is defined as in [2]:

$$
C_{j}=\frac{U_{1}+U_{2}+\ldots+U_{N_{j}}}{B W_{j}},
$$

where $C_{i}$ is the congestion factor at $\mathrm{AP} j$ and $B W_{j}$ is the maximum bandwidth of $54 \mathrm{Mbps}$. Notice that this represents the worst case scenario when all the users are associated with AP $j$. Next, LINGO [7] is used to solve the following ILP [1].

$$
\begin{array}{cc}
\min _{x_{i j}, 1 \leq i \leq N, 1 \leq j \leq M} \max & \left\{C_{1}, C_{2}, \ldots, C_{M}\right\}, \\
\text { subject to } & \sum_{i=1}^{N} x_{i j}=1, \\
& C_{j}=\frac{\sum_{i=1}^{N} U_{i} \cdot x_{i j}}{B W_{j}}, \\
& \text { for } j=1, \ldots, M .
\end{array}
$$

Objective (5.1) minimizes the maximum congestion at the MCAP. Constraint (5.2) states that each user must be assigned to one-and-only-one AP where $x_{i j}$ is a binary variable that is 1 when user $i$ is assigned to AP $j$ and 0 otherwise. The constraint (5.3) defines the congestion factor of AP $j$ depending on the association between users and APs.

Initial assignment of potential candidates is found for all the users using an equally-distributed power level of $17 \mathrm{dBm}$, if the power received by each user is to exceed $-85 \mathrm{dBm}$ and the SIR is greater than 0 dB. A MATLAB algorithm then identifies the MCAP. The MCAP is defined as the AP with the maximum bandwidth utilization as defined in (4). The association matrix is fed to LINGO to optimize the user distribution based on the ILP as specified in (5).

The complete algorithm can be summarized as follows:

Step 1. Assign channels to the $M$ APs by invoking 
LINGO to solve the ILP presented in (2);

Step 2. Randomly distributed users are generated, each with random data rates between 1 Mbps and 7 Mbps. Congestion is then calculated at each AP using (4);

Step 3.Generate a user-association matrix using the propagation model in (1). A user $i$, each with data rate $U_{i}$, may be associated with an $\mathrm{AP} j$ if :

the power received at $i$ from AP $j$ exceeds a threshold value of $-85 \mathrm{dBm}$, the SIR from $\mathrm{AP} j$ at user $i$ is greater than $0 \mathrm{~dB}$.

Step 4. Invoke LINGO to solve the ILP optimization problem using the ILP defined in (5.1), (5.2) and (5.3). A better user-distribution matrix and a better load distribution are now achieved. Based on the current assignment, if the designer is satisfied with the current design, stop. Otherwise, go back to Sep 1.

By current assignment, we mean both channel and user assignments that work most of the time for randomly generated users. Notice the final solution provides a user's association with only one AP at time.

\section{NUMERICAL RESULTS}

Over 20 simulations were run. For clarity of presentation, only one is shown for each scenario, representing the mean performance. In other words, the simulation results provided are neither the worst nor the best case, but somewhere in between. Fig. 1 displays the distribution of APs, users and the power level obtained from the propagation model.

In scenario 1, we created a graphical representation of the power level of the 4 APs, located 60 meters apart and 20 meters away from the walls. Thirty users are randomly placed in a 100 meter length, 100 meter width, and 3 meter height single floor building. The average data rate of users is assumed to be constant throughout the simulation time.

Given the APs' locations and the interference factor between overlapping channels, LINGO is invoked to solve the ILP in (2) for channel assignment. The channels assigned for the 4 APs are 4, 8, 1 and 11 respectively.

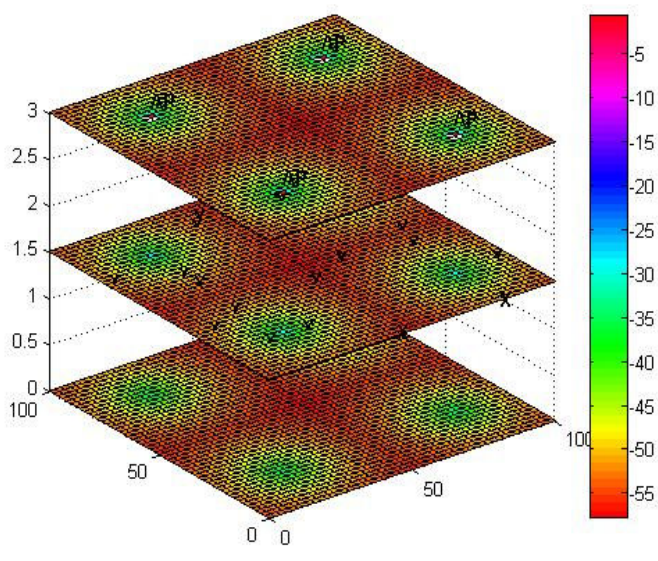

Figure 1: Power level map for scenario 1 at ground level (0 meters), receiver level (1.5 meters), and AP level (3 meters).

We then generated an initial users' association based on a receiver sensitivity value of $-85 \mathrm{dBm}$ and an SIR value greater than $0 \mathrm{~dB} 2$.

Next, LINGO is used to solve the model described in (5) for minimizing the load at the MCAP to produce an optimal user distribution. To illustrate the effectiveness of the proposed algorithm (model 3), the same users' locations, AP's locations and average data rate was applied to two different models; Model 1 uses only receiver sensitivity of -85 $\mathrm{dBm}$ (SIR was not taken into consideration) as a factor in determining the initial associations' matrix [2] whereas model 2 assigns channels to all APs (channel 6) based on the best SIR, inadvertently causing maximum interference among them. It is important to note that in model 1 each user connects to the AP with the highest RSSI.

Similarly, LINGO is invoked to solve the ILP based on the initial associations' matrix obtained by the two different scenarios.

Simulation results demonstrated that our proposed algorithm imposing the SIR threshold is able to distribute the load equitably across the APs. Graphical representation is presented in Fig. 2.

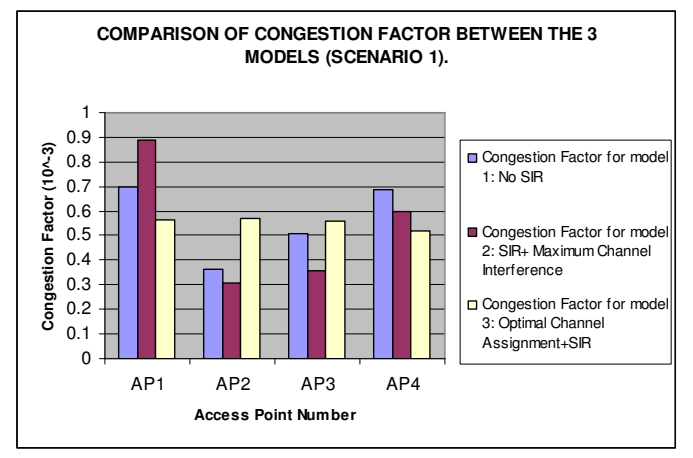

Figure 2: Graphical representation of congestion factor in the 3 models (scenario 1) 
Note that AP1's congestion factor, which was the MCAP (no SIR), was relaxed and its load is shared among other APs. It is very interesting to note that when SIR alone is considered, the network is totally out of balance and users were hardly able to associate with one AP.

Scenario 2 consisted of a grid of 9 APs and 60 users spread out on an area of 160 meters in length, 160 meters in width and 3 meters in height. The distance between APs is kept unchanged. A new random distribution of users with their respective average data rate was generated. LINGO used the ILP in (5) to find the optimal channel assignment for the current configuration. The following channels were assigned to AP1 through AP9, respectively: 11, 4, 8, 1, 11, 4, 11,1 , and 11 .

Results illustrates that our algorithm is able to balance the load and reduce the congestion at the MCAP significantly. A graphical representation of the results is displayed in Fig. 3.

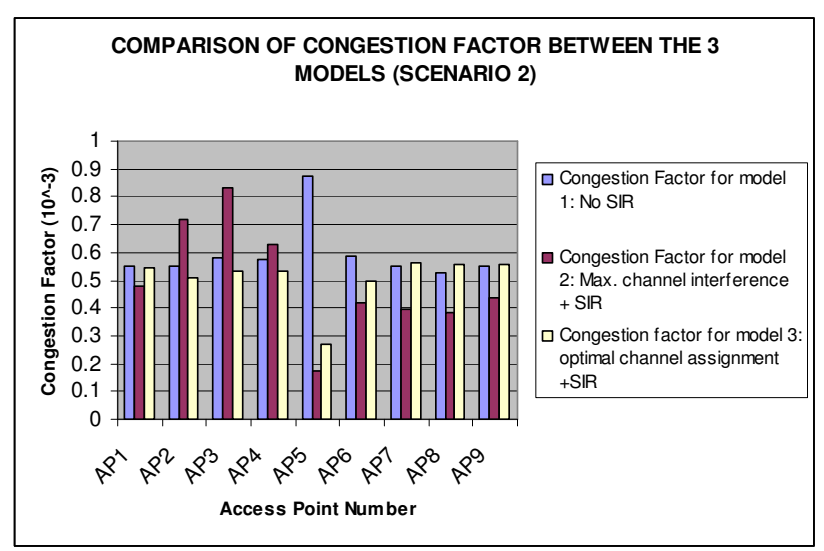

Figure 3: Graphical representation of congestion factor in the 3 models (scenario 2)

We observe that the proposed algorithm enforces a load balance over the WLAN. It is interesting to note that AP5 was the MCAP in model 1 where no interference was accounted for, but with the interference taken into consideration the load on AP5 reduced significantly. However, we note that 14 users out of 60 were not able to associate with any AP on the network in model 2 due to the severe interference level caused on each user. Therefore, we relaxed the SIR constraints on these 14 users $\left(U_{4}, U_{6}, U_{8}, U_{10}\right.$, $U_{18}, U_{27}, U_{31}, U_{37}, U_{39}, U_{41}, U_{47}, U_{49}, U_{55}$ and $\left.U_{59}\right)$ and applied only the receiver constraint of $-85 \mathrm{dBm}$. In other words, each of these users' received power is observed and it was allowed to associate with the AP of the highest RSSI even though its respective SIR was less than $0 \mathrm{~dB}$.

It is important to note that the modeling and simulation solution for the first model was reached within couple of seconds and the solution for the second model was reached within 5-6 minutes. This confirms that the ILP, and the resulting algorithm, are indeed NP-complete.

In conclusion, our computational results suggest that the proposed model can be applied to medium size networks. The resulting design reduces congestion at the MCAP, consonant with the user's locations and their respective interferences from neighboring AP's. Overall, this improves the throughput over the WLAN.

\section{CONCLUSION}

In this paper, a combined algorithm to assign channels to APs, reduce the congestion over the MCAP and balance

the load of users has been proposed. The algorithm extends the model presented in $[2,8]$ to consider SIRs. The algorithm has been shown to provide improved results compared to the no-SIR algorithm described in $[1,2]$. The model has shown to perform well for networks of different topologies. Work is undergoing to extend the model to include dynamic changes in the user's data rate and locations.

Acknowledgment: This research work was funded, in part, by an MRI grant from the National Science Foundation (Grant \#: CNS 0619069).

References
[1] Y. Lee, K. Kim, and Y. Choi, "Optimization of AP placement and channel assignment in wireless LANs," LCN 2002. 27 $7^{\text {th }}$ Annual IEEE Conference on Local Computer Networks. pp. 831-836, November 2002.

[2] R. Akl and S. Park, "Optimal access point selection and traffic allocation in IEEE 802.11 networks," Proceedings of 9th World Multiconference on Systemics, Cybernetics and Informatics (WMSCI 2005): Communication and Network Systems, Technologies and Applications, paper no. S464ID, July 2005.

[3] I. Papanikos and M. Logothetis, "A study on dynamic load balance for IEEE $802.11 \mathrm{~b}$ wireless LAN," Proc. 8th International Conference on Advances in Communication \& Control, COMCON 8, Rethymna, Crete/Greece, June 2001.

[4] H. Velayos, V. Aleo and G. Karlsson, "Load balancing in overlapping wireless LAN cells," Proceedings of IEEE ICC 2004, Paris, France, pp. 3833-3836, June 2004.

[5] J. Lei, R. Yates, L. Greenstein and H. Liu, "Wireless link SNR mapping onto an indoor testbed," Proceedings of IEEE Tridentcom 2005, Trento, Italy,pp. 130-135, February 2005.

[6] O. Brickley, S. Rea and D. Pesch, "Load balancing for QoS optimisation in wireless LANs utilizing advanced cell breathing techniques," unpublished.

[7] L. S. Inc., "LINGO," http://www.lindo.com.

[8] R. Akl and A. Arepally, "Dynamic channel assignment in IEEE 802.11 networks,” IEEE Portable 2007: International Conference on Portable Information Devices, paper no. 1569003128,6 pgs, accepted, March 2007. 